\title{
Baricitinib induces LDL-C and HDL-C increases in rheumatoid arthritis: a meta- analysis of randomized controlled trials
}

Chengfeng Qiu ${ }^{1,2+}{ }^{+}$Xiang Zhao ${ }^{3 \dagger}$, Lang She ${ }^{2}$, Zhihua Shi ${ }^{2}$, Ziwei Deng ${ }^{2}$, Liming Tan ${ }^{4}$, Xiaojun Tu', Shilong Jiang ${ }^{5}$ and Bin Tang ${ }^{1 *}$ (D)

\begin{abstract}
Background: Baricitinib, an oral-administrated selective inhibitor of the JAK1 and JAK2, is recently approved for rheumatoid arthritis (RA) treatment. With the aim to provide some insights on the clinical safety, the current study mainly focused on the effect of baricitinib on low-density lipoprotein cholesterol (LDL-C) and high-density lipoprotein cholesterol (HDL-C) levels and cardiovascular risk.

Methods: The net change scores [least squares mean (LSM) and mean change] of LDL-C and HDL-C levels from baseline with the comparison of baricitinib versus placebo were pooled, respectively. Risk rations (RR) of major cardiovascular events (MACEs) and differences of cardiovascular risk scores at the end of treatment across groups were compared.

Results: Six trials with randomized 3552 patients were finally included in summary analysis. Results showed that baricitinib significantly increased LDL-C levels, the net mean change was $13.15 \mathrm{mg} / \mathrm{dl}$ with 95\% Cl 8.89 17.42 $\left(\mathrm{I}^{2}=\right.$ 0 ) and the net LSM was $11.94 \mathrm{mg} / \mathrm{dl}$ with 95\% Cl 7.52 16.37 ( $\left.\mathrm{I}^{2}=84 \%\right)$. HDL-C also increased obviously with the net LSM change was $7.19 \mathrm{mg} / \mathrm{dl}\left(95 \% \mathrm{Cl}, 6.05 \sim 8.33, \mathrm{I}^{2}=47 \%\right)$ and net mean change was $5.40 \mathrm{mg} / \mathrm{dl}(95 \% \mathrm{Cl}, 3.07 \sim 7.74$, $\left.\mathrm{I}^{2}=10 \%\right)$. Subgroup and meta-regression analysis demonstrated baricitinib induced LDL-C and HDL-C increases in a dose-response manner. However, both the pooled RRs of MACEs and differences of cardiovascular risk scores were not statistically significant across groups.
\end{abstract}

Conclusion: This study confirmed that baricitinib induced a stable dose-response increase in LDL-C and HDL-C levels. Since the causality association between altered lipids and cardiovascular risk was not identified yet, this issue cannot be completely dismissed. Future research is needed to fully dissect the implications of these lipid changes.

Keywords: Baricitinib, Rheumatoid arthritis, Low-density lipoprotein cholesterol, High-density lipoprotein cholesterol, Cardiovascular risk

\section{Background}

Rheumatoid arthritis (RA) is a chronic, systematic autoimmune disease characterized by synovial inflammation and joint damage [1]. For a long time, conventional synthetic disease-modifying antirheumatic drugs (cDMARDs), usually methotrexate (MTX), ranked as the frontline therapy for RA targeting to improve synovitis and physical

\footnotetext{
* Correspondence: huaihuatangbin@163.com

${ }^{+}$Chengfeng Qiu and Xiang Zhao are equal responsibility for this article.

'Departement of Evidence-base Medcine and Clinical center, The First

People's Hospital of Huaihua of University of South China, Huaihua 418000,

People's Republic of China

Full list of author information is available at the end of the article
}

function [2]. However, many patients do not achieve the therapy goal, failing to continue therapy because of seriously adverse effect or lose response over time. These issues highlight the need to develop additional therapeutic strategies for RA. Recent advances put emphasis on the crucial role of cytokine network involved in the pathogenesis of RA [2]. Janus kinases (JAKs) are part of the tyrosine kinases family that regulate a variety of signaling cascades of cytokine, many of which are contributed to the RA progression. A new class of small molecules targeting JAKs are successfully developed to be an additional therapy for RA patients [3].

(C) The Author(s). 2019 Open Access This article is distributed under the terms of the Creative Commons Attribution 4.0 International License (http://creativecommons.org/licenses/by/4.0/), which permits unrestricted use, distribution, and 
Baricitinib is an oral-administrated selective inhibitor of the JAK1 and JAK2. As the second JAK inhibitor, baricitinib recently attracted numerous attention for the approval of RA treatment in European Union, Japan and USA [4]. Completed phase II and III trials demonstrated baricitinib significantly improves symptoms of moderate-to-severe active RA patients who had undergone an inefficient response or intolerance to cDMARDs, presenting a therapy value of baricitinib in a new field of RA treatment [5].

RA is strongly associated with high risk of cardiovascular $(\mathrm{CV})$ events. A pooled analysis demonstrated the $\mathrm{CV}$ risk was $48 \%$ higher in patients with RA than those in general individuals [6]. The mechanism underlying the excess risk of $\mathrm{CV}$ events in RA remains unclear, but the traditional risk factors, systematic inflammation and the other RA-specific (mainly the disease activity) may play a role in this increased risk [7]. Particularly, recognition of $\mathrm{CV}$ risk of anti-rheumatism treatment was important to guide the clinical management of RA. Both MTX and tumor necrosis factor (TNF) inhibitor (infliximab) are associated with decreased CV risk [8, 9]. The cardiovascular risk associated with non-steroidal anti-inflammatory drugs (NSAIDs) was modest lower than in non-RA individuals [10]. As a new class of agent for RA, the safety of baricitinib, especially their effects on cardiovascular risk and associated risk factors, remains to be assessed. For now, the follow-up duration of completed phase II and III trials were 12 52 weeks. Such short follow-up duration is inappropriate for assessment of $\mathrm{CV}$ risk. A long-term observation is deserving to be held and the associated risk should be estimated.

Dyslipidemia, especially the elevated low-density lipoprotein cholesterol (LDL-C) levels which is regarded as "bad cholesterol", has been well established as the major risk of $\mathrm{CV}$ disease $[11,12]$. In contrast, serum high-density lipoprotein cholesterol (HDL-C) is considered to be protective against cardiovascular disease with the name of "good cholesterol" [11, 12]. With the aim to provide some insights on the clinical safety of baricitinib, the current study mainly focuses on the effect of baricitinib with different dose on LDL-C and HDL-C levels and potential cardiovascular risk.

\section{Materials and methods}

We followed the guidelines proposed by the Cochrane handbook for performing and reporting the current meta-analysis [13].

\section{Search methods and resources}

Studies that reported the effect of baricitinib on plasma lipids in patients with rheumatoid arthritis were considered as our interest. Two investigators independently searched the relevant studies across these databases:
Medline, Embase, and the Cochrane Central Register of Controlled Trials (CENTRAL) and websites (www.clinicaltrials.gov). The following key words were used for searching: (JAK inhibitor OR baricitinib OR LY3009104 OR INCB028050) AND ("rheumatoid arthritis" OR RA). The details of search algorithm of Medline (Via PubMed) was provided in Additional file 1. In addition, we also extend the search by scrutinizing the reference lists from all relevant studies or reviews. The last study search was updated in 1 October 2018.

\section{Study selection and data extraction}

Two investigators (She L and Jiang SL) independently identified eligible trials by carefully scrutinized titles, abstracts and full text. Consensus was achieved through consulted with the third investigator in case of disagreement. Original studies were considered as eligible if they meet the pre-defined inclusion criteria: (i) patients were 18 years of age or older and had active rheumatoid arthritis; (ii) the comparation were the baricitinib versus placebo or active control with or without any background therapy; (iii) reported outcomes include the change scores of LDL-C and HDL-C, the estimation of potential cardiovascular risk at the end of treatment; (iv) clinical trials of randomized, double-blind, placebo- or active-controlled design. Of note, any studies that with duplicated reporting, important information missing or failed to meet any of the inclusion criteria listed above were excluded.

Two investigators (She L and Jiang SL) extracted the following data independently from the primary text of individual trial: trial name, recruitment period, number of centers, publication year, patients' demographics and clinical characteristics, follow-up duration, duration of $\mathrm{RA}$, randomized patients, background therapy, the change values of LDL-C and HDL-C at the end of treatment from baseline.

\section{Outcomes}

In this study, we focused on the net change of LDL-C and HDL-C levels at the end of follow-up over baricitinib treatment when compared to the placebo or the active-control. Net change scores were expressed as the net least squares mean (LSM) and net mean change at the end of follow-up from baseline, the two scores were pooled separately in the current meta-analysis. Net mean change scores were calculated as: (measure at end of follow-up in the treatment group-measure at baseline in the treatment group) - (measure at end of follow-up in the control group-measure at baseline in the control group). LSM means adjusted mean in primary text, and is apparently more reasonable because the potential confounders have been adjusted. In additional, with the aim to assess the potential cardiovascular risk of baricitinib, 
we calculated the risk of MCVEs and the differences of $\mathrm{CV}$ scores with the comparations of baricitinib versus placebo or versus active agents. Formulas used for data transformation were listed in Additional file 2.

\section{Bias risk assessment}

Two investigators (Deng ZW and Shi ZH) judged the bias risk and quality of included trials by using Cochrane risk of bias assessment tool independently. According to the guidelines, five aspects were assessed: allocation sequence generation, allocation concealment, blinding of participants and investigators, completeness of outcome data, and selective outcome reporting.

\section{Statistical analysis}

In this meta-analysis, we compared the net changes of serum LDL-C and HDL-C levels at the end of treatment from baseline in baricitinib group to those in control group. Net LSM change and net mean change of LDL-C and HDL-C levels were pooled using the DerSimonian-Laird random-effects model, respectively. For studies containing multiple intervention with different dose, we combined them to create a single pairwise comparison by using a weighted average [13]. Pooled effect sizes were represented as weight difference (WMD) and $95 \%$ confidence interval $(\mathrm{CI})$. To estimate the CV risk, the $\mathrm{CV}$ risk scores (Framingham risk score and Reynolds risk score) of patients with different treatment were compared and numbers of MACEs in each group were extracted to calculate risk rations (RRs). In order to assess the robustness of pooled results and identify the potential contributor of heterogeneity, sensitivity analysis was conducted using the leave-one-out method in each turn to investigate the influence of single study on the overall risk estimate. In view of dose-dependent and time-dependent clinical effect with most of drugs, we performed a subgroup analysis and a random-effects meta-regression to evaluate the association between baricitinib-induced elevation of plasma LDL-C and HDL-C levels with treatment dose and duration.

Between-study heterogeneity was quantitatively assessed using the $\mathrm{I}^{2}$ index [14], values of 25,50 , and $75 \%$ presented as mild, moderate, and high heterogeneity. Potential publication bias was explored by visual inspection of funnel plots for asymmetry [15] and statistical evaluation with Begg's rank correlation test [16] and Egger's linear regression test [17]. Two-tailed $\alpha$ level of significance was set at 0.05 .

All statistical analyses were performed with Review Manager Version 5.3 (The Nordic Cochrane Center, Copenhagen, Denmark) and STATA/SE.12.0 (StataCorp, College station, Texas, USA).

\section{Results}

Baseline characteristics of included trials

After a critically reviewed the title, abstract and full text according to the predefined criteria, six trials were finally included in the current meta-analysis, including four III phases trials (BRA-BUILD, RA-BEGIN, RA-BEACON and RA-BEAM) and two II phases trials (NCT01469013 and NCT01185353). Flow diagram of study selection process is presented in Fig. 1. Except one trial performed as single center study [18], all the rest are multicenter studies. A total of 3552 RA patients were randomized into 5 arms: control, and baricitinib with different dose ( $1 \mathrm{mg}, 2 \mathrm{mg}, 4 \mathrm{mg}$ and $8 \mathrm{mg}$ ). Among them, $78 \%$ of patients were female $(n=2788)$. Except one trial that included the patients with a short RA duration (ranged from 1.3 1.9 years) and receiving no prior DMARDs therapy, the rest patients had a long RA duration (duration of RA $\geq 5$ years) and has undergone an insufficient response or intolerance to $\geq 1$ DMARDs. Most patients $(n=2964)$ have added a stable background therapy including any DMARDs in addition to the placebo or baricitinib, there are 588 patients who received no background therapy. Follow-up duration ranged from 12 52 weeks. More details of study characteristics were presented in the Table 1, and the characteristics of included studies for analysis of other JAK inhibitors were displayed in Additional file 3.

\section{Baricitinib induces LDL-C and HDL-C increases in patients with RA}

To systematically and completely evaluate the effect of baricitinib on LDL-C, we compared net change scores of LDL-C levels in patients with baricitinib treatment to this in patients with placebo (Fig. 2a). Pooled results showed that baricitinib significantly increased the levels of serum LDL-C after treatment for 6 52 weeks regardless of the previous treatment (DMARDs-naïve or resistant) when compared with placebo, the net mean change was $13.15 \mathrm{mg} / \mathrm{dl}\left(95 \% \mathrm{CI}, 8.89 \sim 17.42, \mathrm{I}^{2}=0\right)$, even adjusted the potential confounders, the net change scores which presented as net LSM remained significantly (net LSM, $11.94 \mathrm{mg} / \mathrm{dl}$, 95\% CI, 7.52 16.37, $\mathrm{I}^{2}=84 \%$ ).Similar to LDL-C, HDL-C increased obviously in RA patients seen after baricitinib treatment (Fig. 2b), with the net LSM change was $7.19 \mathrm{mg} / \mathrm{dl}\left(95 \% \mathrm{CI}, 6.05 \sim 8.33, \mathrm{I}^{2}=\right.$ $47 \%)$ and net mean change was $5.40 \mathrm{mg} / \mathrm{dl}(95 \% \mathrm{CI}$, $3.07 \sim 7.74, \mathrm{I}^{2}=10 \%$ ). Sensitivity meta-analysis showed these pooled results of net LSM change of LDL-C (Additional file 4: A) and HDL-C (Additional file 4: B) were robust with no significant change by any single study, but the heterogeneities have greatly reduced after excluded one study, while the $\mathrm{I}^{2}$ reduced to $52 \%$ from $84 \%$ in the estimation of LDL-C change and the $\mathrm{I}^{2}$ reduced to 0 from $47 \%$ (Additional file 5). 


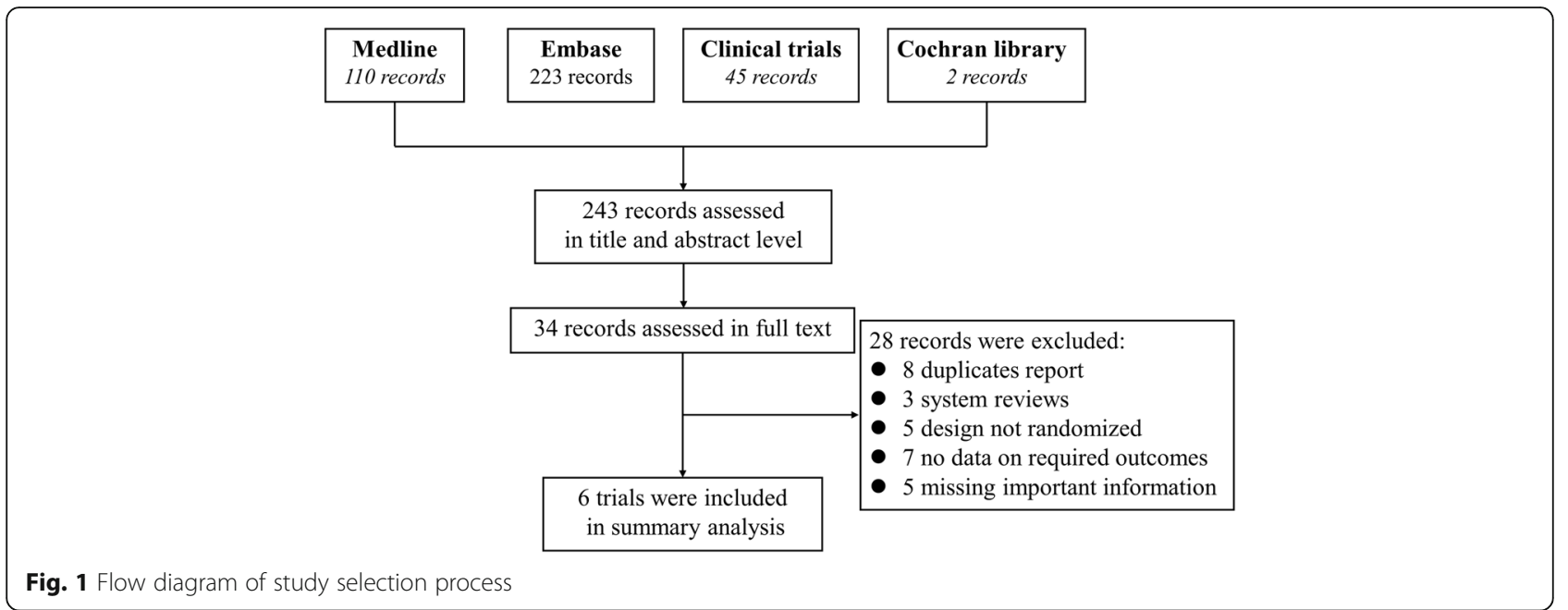

In additional, we also estimated the effect of the other JAK inhibitors on LDL-C (Additional file 6) and HDL-C levels (Additional file 7) in patients with RA. Summary data showed that tofacitinib (pan-JAK inhibitor) and decernotinib (selective JAK3 inhibitor) had much stronger effect on LDL-C increase with the net mean change of $16.84 \mathrm{mg} / \mathrm{dl}(95 \% \mathrm{CI}, 10.31 \sim 23.36)$ and $15.44 \mathrm{mg} / \mathrm{dl}$ (95\% CI, 6.58 24.31), respectively. Filgotinib (selective JAK1 inhibitor) moderately increased LDL-C level with the net mean change of $9.59 \mathrm{mg} / \mathrm{dl}$ (95\% CI, 3.61 15.57). Surprisingly, results demonstrated peficitinib (mainly moderately inhibits JAK3) did not have obvious effect on LDL-C levels (net mean change, $0.77 \mathrm{mg} / \mathrm{dl}, 95 \% \mathrm{CI}$, 0.47 2.01). As for the assessment of HDL-C levels, the net mean changes for tofacitinib, decernotinib and filgotinib were $8.09 \mathrm{mg} / \mathrm{dl}$ (95\% CI, 5.40 10.79), $3.25 \mathrm{mg} / \mathrm{dl}$ (95\% CI, 1.00 7.51) and 4.24 mg/dl (95\% CI, 0.69 7.78), respectively. Of note, peficitinib significantly increased HDL-C levels (net mean change, $6.64 \mathrm{mg} / \mathrm{dl}, 95 \% \mathrm{CI}$, 0.72 12.57), other than its effect on LDL-C.

\section{Baricitinib-induced increases of LDL-C and HDL-C are mainly associated with treatment dose}

Baricitinib was recently approved for RA treatment. However, baricitinib $4 \mathrm{mg}$ was not permitted in USA now. In this section, we mainly focused on the potential dose- and time -dependent response on LDL-C and HDL-C levels. Figure $3 \mathrm{a}$ and $\mathrm{b}$ presented the LSM change of LDL-C and HDL-C for placebo, baricinitib 2 $\mathrm{mg}$ and $4 \mathrm{mg}$ at the week $12,24,52$, respectively. It seemed that the increased LDL-C and HDL-C levels which induced by baricitinib were more related to treatment dose than that related to treatment duration. We further performed a subgroup analysis of LDL-C change (Fig. 3c) and HDL-C change (Fig. 3d) stratified by treatment duration and treatment dose. We observed that the net LSM changes of LDL-C for baricinitib $2 \mathrm{mg}$ and
$4 \mathrm{mg}$ compared to placebo was increased as the treatment duration was extended (12-week to 52-week), however, the net LSM change of HDL-C remained stable across baricinitib $2 \mathrm{mg}$ and $4 \mathrm{mg}$ from week 12 to week 52.

When compared to placebo, the net change scores of LDL-C (Fig. 3c) and HDL-C (Fig. 3d) for baricinitib 4 mg was much higher than that for baricinitib $2 \mathrm{mg}$ not only at week 12 but also at week 24 . We further compared baricitinib $4 \mathrm{mg}$ with $2 \mathrm{mg}$, net LSM change of LDL-C was $4.34 \mathrm{mg} / \mathrm{dl}(95 \% \mathrm{CI},-2.34 \sim 11.03)$ and 5.74 $\mathrm{mg} / \mathrm{dl}$ (95\% CI, 0.16 11.32) at week 12 and week 24, and the net mean change of LDL-C was $2.36 \mathrm{mg} / \mathrm{dl}$ (95\% CI, -5.36 10.08) at week 12; net LSM change of HDL-C was $1.58 \mathrm{mg} / \mathrm{dl}$ (95\% CI,0.04 3.12) and $2.85 \mathrm{mg} /$ dl (95\% CI,0.96 4.75) at week 12 and week 24, and the net mean change of HDL-C was $4.20 \mathrm{mg} / \mathrm{dl} \quad(95 \%$ CI,-0.02 8.42) at week 12 .

Random-effects meta-regression suggested a significant association between baricitinib-induced elevation of LDL-C and HDL-C level with treatment dose [LDL-C (Fig. 4a): adjusted $R^{2}=23.6 \%$, slope $=7.01,95 \%$ CI, 1.64 12.38; HDL-C (Fig. 4b): adjusted $\mathrm{R}^{2}=34.64 \%$, slope $=5.17,95 \% \mathrm{CI}, 2.92 \sim 7.43)]$. But no significant association was found between LDL-C and HDL-C elevation with duration of treatment (data not show).

\section{Cardiovascular risk assessment of baricitinib treatment in patients with RA}

Aimed to assess the $\mathrm{CV}$ risk of baricitinib treatment, first, we calculated the risk of MACE in patients with baricitinib treatment compared to placebo or the other active agents (MTX and adalimumab). As showed in Fig. $5 \mathrm{a}$, pooled RR was 1.08 with $95 \%$ CI 0.10 to 11.42 (baricitinib vs. placebo), 3.03 with $95 \%$ CI 0.28 to 33.19 (baricitinib vs. MTX) and 0.74 with $95 \%$ CI 0.07 to 8.10 (baricitinib vs. adalimumab), but no statistically 


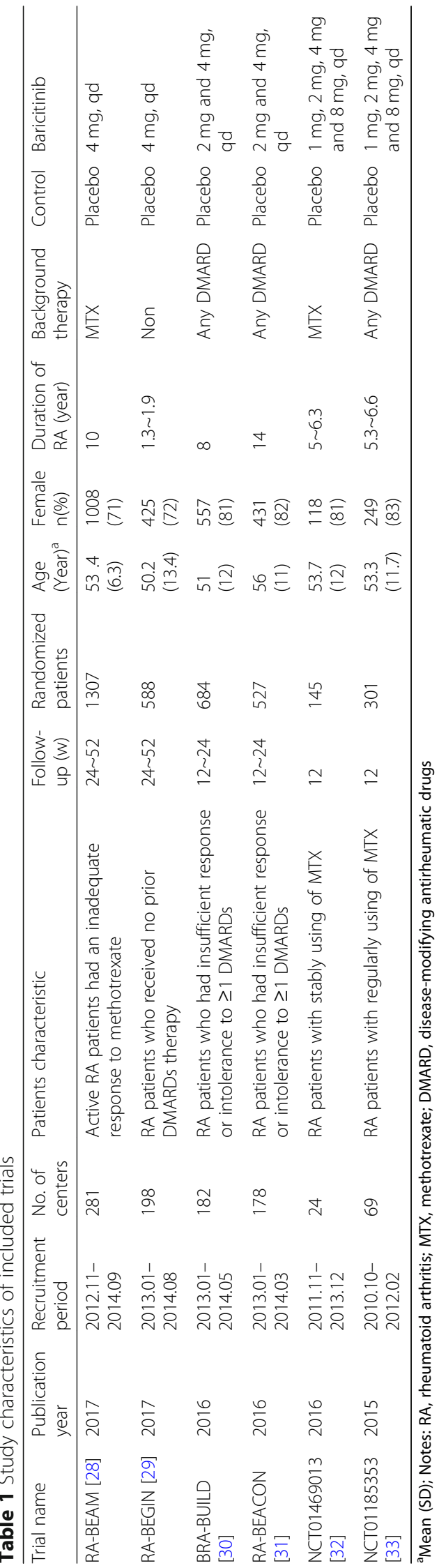




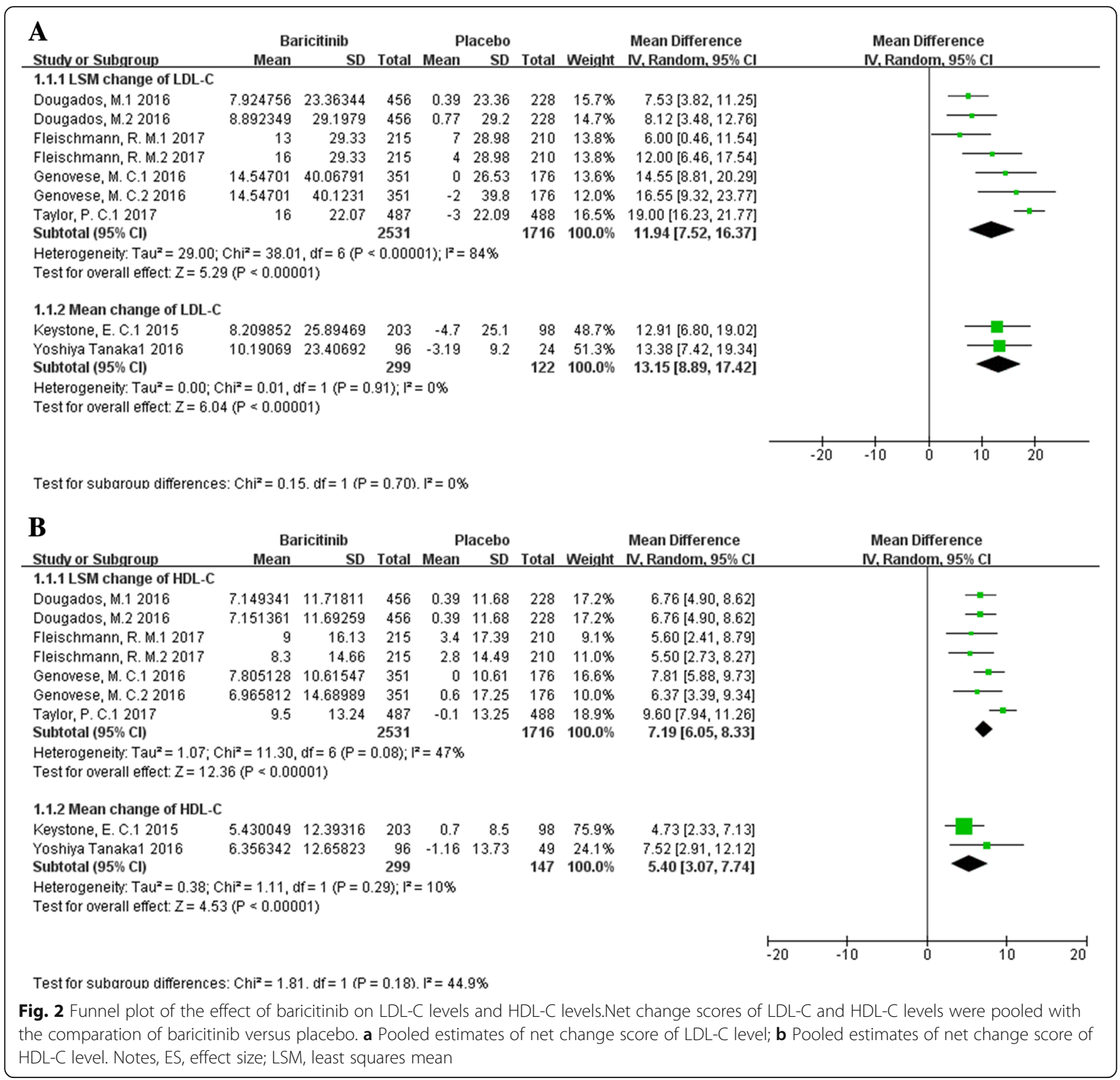

significant was found across all comparations. Furthermore, we observed no significant differences of CV risk scores at week 12 (both for Framingham risk score and Reynolds risk score) between the baricitinib and placebo group (Fig. 5b).

\section{Bias assessment}

Overall risk of bias was ranked as low in included trials (Additional file 8). There was no obvious publication bias was assessed by visual inspection of funnel plots for asymmetry (Fig. 6) and through Begg's rank correlation test ( $p$ values for LDL-C and HDL-C estimation were $0.36,0.17$, respectively) and Egger's linear regression test
( $\mathrm{p}$ values for LDL-C and HDL-C estimation were 0.34 , 0.07 , respectively).

\section{Discussion}

The salient findings of this meta-analysis of 6 randomized controlled trials (RCTs) including 3552 randomized patients with RA can be listed as follows. First, baricitinib treatment regardless of $2 \mathrm{mg}$ and 4 mg significantly induces LDL-C and HDL-C increases in patients with RA when compared with placebo both at week 12, 24 and 52. Second, baricitinib-induced increased in LDL-C and HDL-C were strongly associated with the treatment dose but not with the treatment duration, suggesting a dose - 

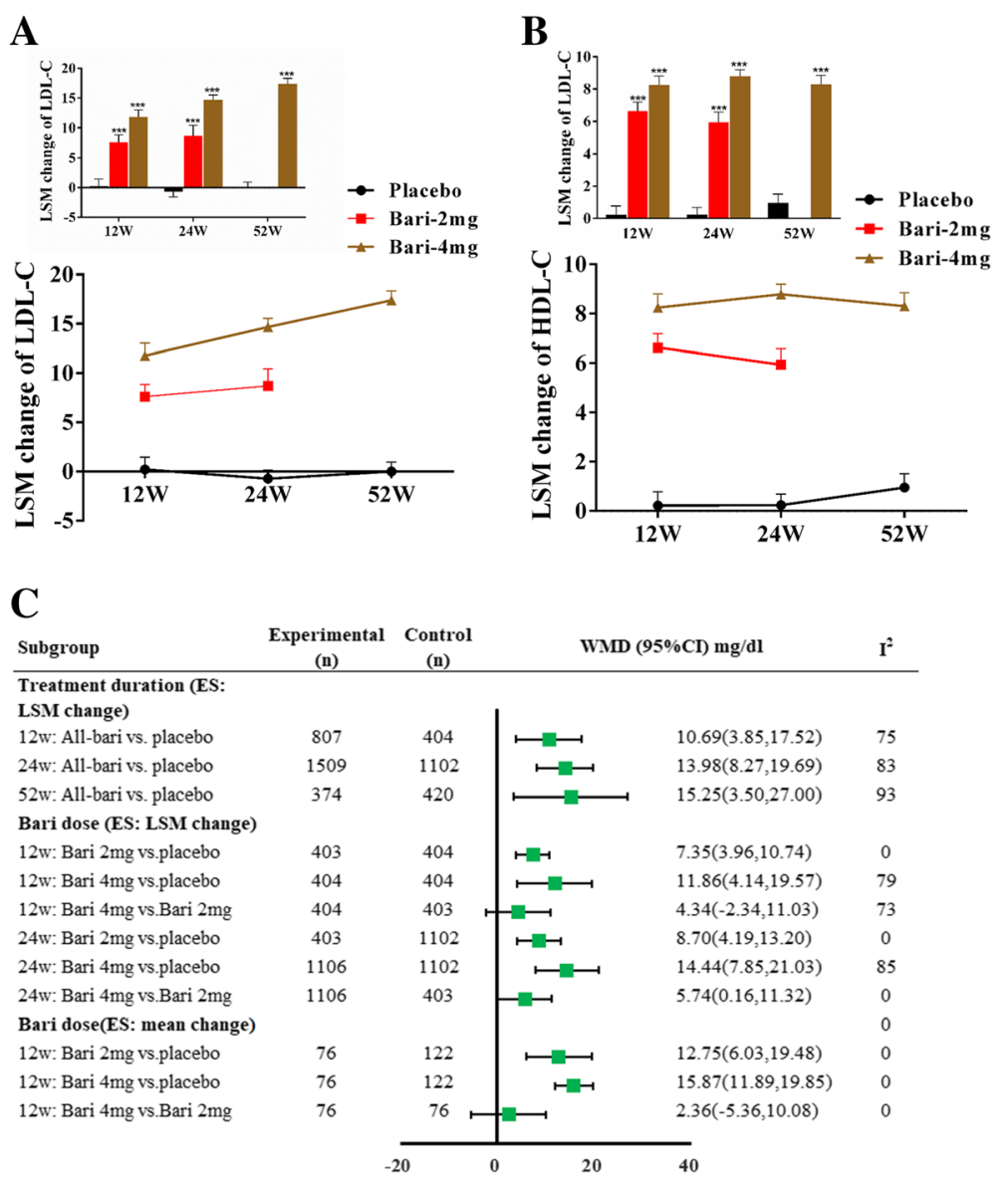

\begin{tabular}{|c|c|c|c|c|c|}
\hline Subgroup & $\begin{array}{l}\text { Experimental } \\
\text { (n) }\end{array}$ & $\begin{array}{c}\text { Control } \\
\text { (n) }\end{array}$ & WMI & $\%$ CI) $\mathrm{mg} / \mathrm{dl}$ & $I^{2}$ \\
\hline M change) & & & & & \\
\hline 12w: All-bari vs. placebo & 807 & 404 & He- & $7.27(5.93,8.60)$ & 0 \\
\hline 24w: All-bari vs. placebo & 1509 & 1102 & 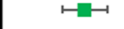 & $7.26(5.31,9.21)$ & 67 \\
\hline 52w: All-bari vs. placebo & 374 & 420 & $\longmapsto$ & $7.24(4.91,9.57)$ & 46 \\
\hline Bari dose (ES: LSM change & & & & & \\
\hline 12w: Bari 2mg vs.placebo & 403 & 404 & 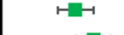 & $6.48(4.94,8.02)$ & 0 \\
\hline 12w: Bari 4mg vs.placebo & 404 & 404 & $H-1$ & $8.05(6.51,9.60)$ & 0 \\
\hline $12 \mathrm{w}$ : Bari $4 \mathrm{mg}$ vs.Bari $2 \mathrm{mg}$ & 404 & 403 & F-1 & $1.58(0.04,3.12)$ & 0 \\
\hline 24w: Bari $2 \mathrm{mg}$ vs.placebo & 403 & 1102 & $\mapsto$ 늘-1 & $5.69(3.89,7.49)$ & 0 \\
\hline 24w: Bari 4mg vs.placebo & 1106 & 1102 & 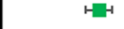 & $8.54(7.38,9.70)$ & 2 \\
\hline 24w: Bari 4mg vs.Bari 2mg & 1106 & 403 & $15-1$ & $2.85(0.96,4.75)$ & 0 \\
\hline Bari dose(ES: mean change & & & & & 0 \\
\hline 12w: Bari 2mg vs.placebo & 76 & 122 & $\longrightarrow$ & $2.36(-0.99,5.70)$ & 0 \\
\hline 12w: Bari 4mg vs.placebo & 76 & 122 & 以— & $6.57(3.19,9.95)$ & 0 \\
\hline $12 \mathrm{w}$ : Bari $4 \mathrm{mg}$ vs.Bari $2 \mathrm{mg}$ & 76 & 76 & $\longrightarrow$ & $4.20(-0.02,8.42)$ & 0 \\
\hline
\end{tabular}

Fig. 3 Subgroup analyses sorted by treatment dose and duration. $\mathbf{a}$ and $\mathbf{b}$ LSM changes of LDL-C and HDL-C levels across placebo, baricitinib $2 \mathrm{mg}$ and $4 \mathrm{mg}$ from week 12 to week 24 to baseline. Values presented as LSM \pm SE. vs. placebo, ${ }^{* * *} p<0.001$; c Subgroup analysis of LDL-C changescores; d Subgroup analysis of HDL-C change scores. Notes, Bari, baricitinib; WMD, weighted mean difference; LSM, least squares mean. SE, standard error

response manner of baricitinib in inducing LDL-C and HDL-C increases. Third, there was no significant differences of $\mathrm{CV}$ risk between baricitinib and placebo groups during the follow-up of 52 weeks.
Patients with RA are strongly associated with increased risk of $\mathrm{CV}$ disease which could hardly be fully explained by traditional risk factors [6]. Further adding to the confusion, active RA present a fall in both LDL-C 

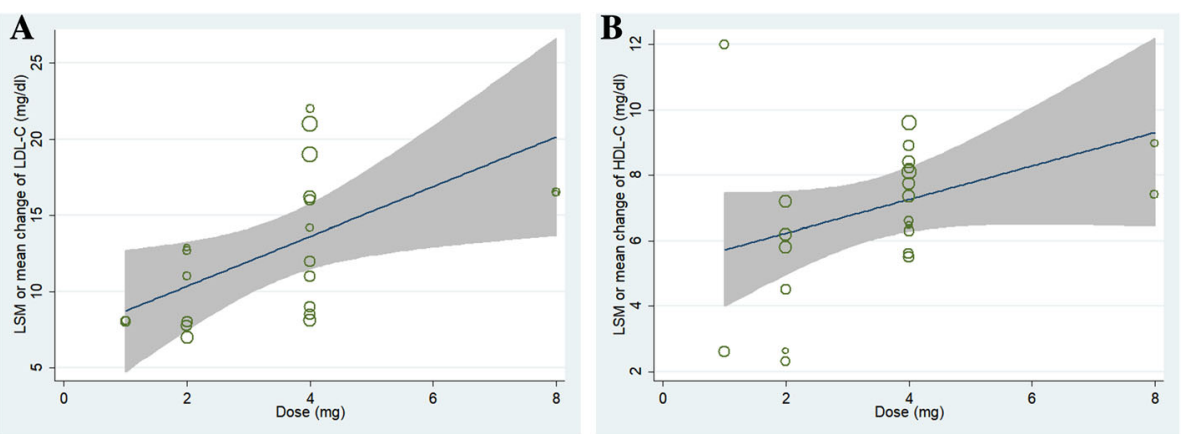

Fig. 4 Meta-regression of the association between baricitinib dose and elevated LDL-C (a) and HDL-C levels (b)

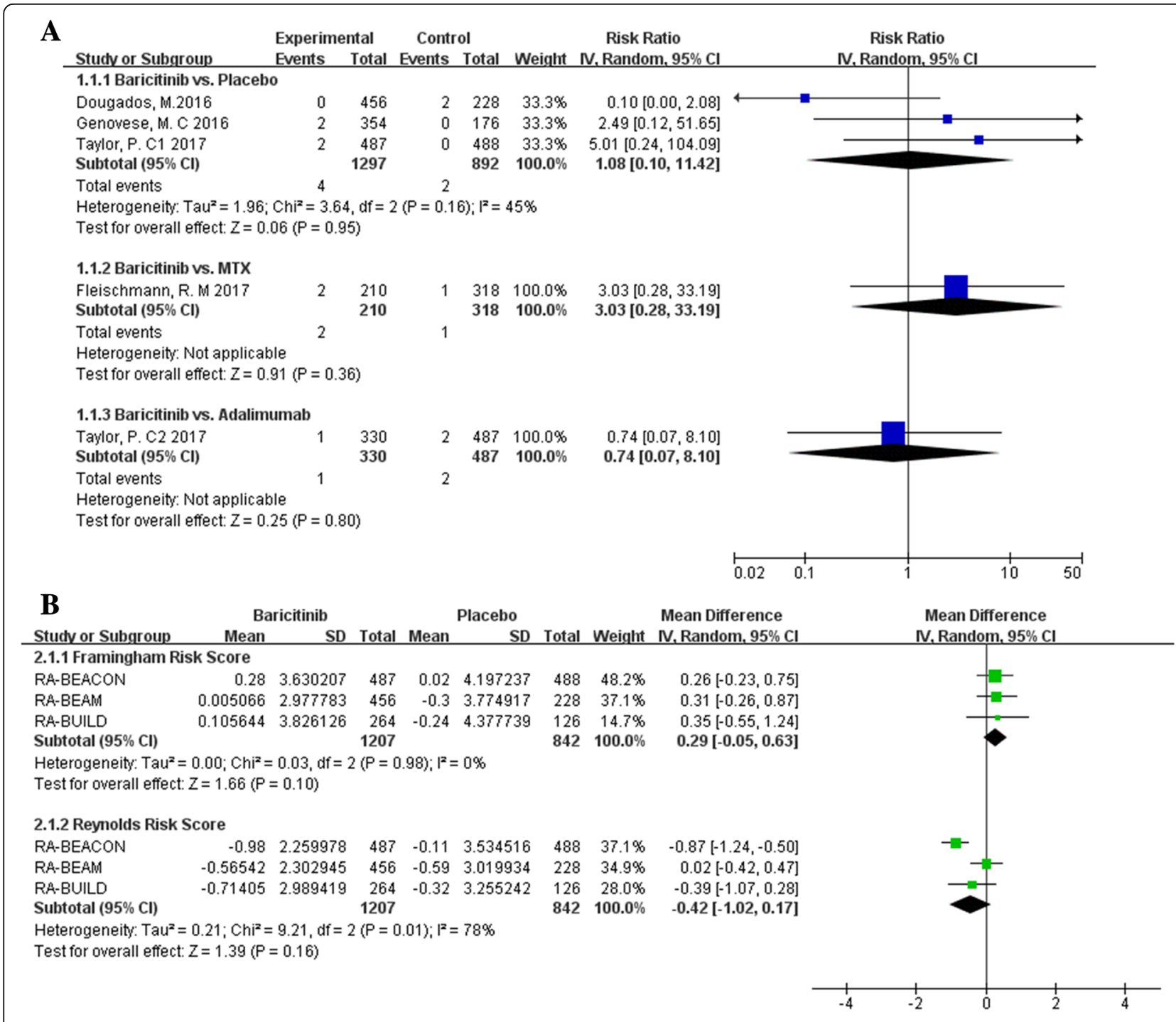

Fig. 5 Pooled estimates of the relative risk of the incidence of major cardiovascular events (a) and the differences of cardiovascular risk scores (b) 


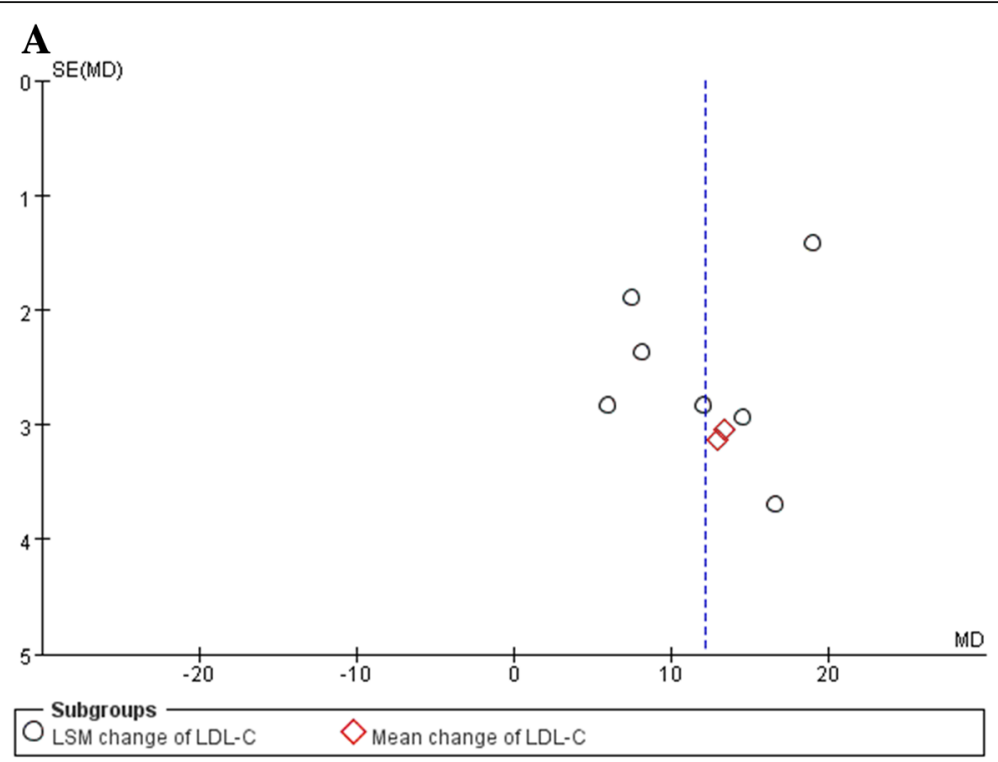

B

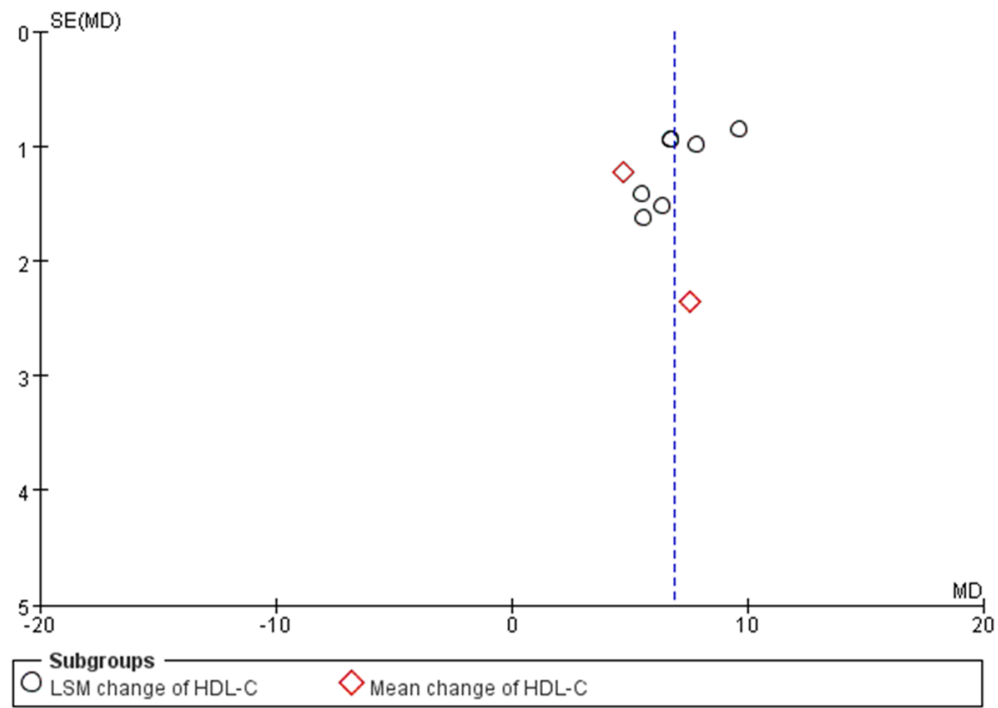

Fig. 6 Funnel plot of baricitinib and LDL-C level (a) and HDL-C level (b)

and HDL-C levels which called "lipid paradox"- decreased lipids and increased CV risk [19]. Systemic inflammation is proposed to play a role in the increased $\mathrm{CV}$ risk and also in the altered lipid metabolism associated with RA [19]. Anti-inflammatory therapy with TNF $\alpha$-inhibitor (adalimumab) induced an elevation of LDL-C and HDL-C mildly seen after treatment, confirming the potential role of inflammation in lipid metabolism [20]. Results of the current study also showed that various JAK inhibitors except to peficitinib all lead to an elevation both of LDL-C and HDL-C levels. Of note, these increases induced by JAK inhibitors were much higher than those induced by adalimumab, studies also demonstrated that adalimumab-induced lipids level is transient [21] while JAK inhibitors-induced LDL-C and
HDL-C elevation lasted for the full period of treatment, these results suggesting that suppression of inflammation just partially underlies the increases in lipids levels, factors specific to different treatment may have a strong influence on the degree and pattern of lipid profile change. Even across the various JAK inhibitors, the change levels of LDL-C and HDL-C were much different from each other. Among them, tofacitinib (a pan-JAK inhibitor), the first JAK inhibitor, had the strongest increases both in LDL-C and HDL-C levels. Baricinitib (inhibitor of JAK1 and JAK2), newly licenced for the treatment of RA, also lead to significant elevation of LDL-C and HDL-C. Surprisingly, peficitinib, moderate selectivity for JAK-3 inhibition, obviously increased HDL-C level but had no change in LDL-C levels, 
providing some additional insight for understanding the role of different JAK signaling in cholesterol metabolism. In additional, the activation of JAK signaling are demonstrated that involved in the pathological process of many cancers [22, 23]. Epidemiological studies showed that lipids level including total cholesterol (TC), LDL-C and HDL-C all much lower in these patients with cancer than those in general population [24]. Though these sighs could not confirm the direct link between JAK activation and lower levels of lipids, this information have raised our awareness regarding whether JAK signaling pathway involved in cholesterol metabolism.

Baricinitib, a selectively inhibitor for JAK1 and JAK2, has been approved for RA treatment in European Union and Japan in 2017 [4]. Recently, considering the insufficient clinical safety data, just baricinitib $2 \mathrm{mg}$ but not $4 \mathrm{mg}$ was approved for RA treatment in USA. In the current study, we explored the dose-response of baricinitib on lipids changes, both subgroup studies stratified by dose and meta-regression all demonstrated consistent results that baricinitib lead to a stable dose-dependent increases in LDL-C and HDL-C levels. Particularly, baricinitib-induced LDL-C change gradually increased as the extended duration of treatment (week 12 to 52), however, the HDL-C increase was similar at the point of week 12, 24 and 52, suggesting the LDL-C elevation was associated with baricinitib dose and treatment duration but HD-C elevation was mainly associated with dose.

$\mathrm{RA}$ is an independent risk factor for CV disease [25]. However, the following are some questions needing evaluation in the changed lipids and $\mathrm{CV}$ risk field: (i) whether the increased LDL-C level further increased the risk of $\mathrm{CV}$ disease in patients with RA; and (ii) whether the increased HDL-C perform a protective role against $\mathrm{CV}$ disease. Particularly, it should also be mentioned that CV risk is not only dependent on a particular cholesterol level, but also is strongly depend on the composition of lipoporteins [26], which is not routinely analyzed in daily clinical practice. Here we compared the risk of MACE at the end of treatment, though there was no significant differences across the baricitinib, MTX and adalimumab, the result should be interpreted with caution because of short follow-up duration, which is limited to observe the incidence of $\mathrm{CV}$ events. Furthermore, we calculated the net change of Framingham and Reynolds $\mathrm{CV}$ risk scores at week 12 to estimate the $\mathrm{CV}$ risk. Pooled results also displayed no significant difference with the comparation of baricitinib versus placebo. Actually, CV risk is underestimated in the patients with RA using these calculators [27], indicating that the CV risk estimation based on the current available studies could hardly reflect the reality. In this respect, long-term observational data will be important across the field.
Some limitations of the current meta-analysis should be recognized. First, though the stable of dose-response of baricitinib on LDL-C and HDL-C levels was confirmed, the underlying mechanism remains further study. Second, our study was not powered to identify the relationship between baricitinib-induced lipid changes and $\mathrm{CV}$ risk. Third, the estimation of $\mathrm{CV}$ risk of baricitinib remains to be further confirmed with long-term observation.

\section{Conclusions}

This study confirmed that baricitinib induced a stable dose-response increase in LDL-C and HDL-C levels. Since the causality association between altered lipids and $\mathrm{CV}$ risk was not identified, however, this issue cannot be completely dismissed. Further research is needed to fully dissect the consequences of these lipid changes and how baricitinib modulate cholesterol metabolism.

\section{Additional files}

Additional file 1: Formulas used in the current study. (DOCX $16 \mathrm{~kb}$ )

Additional file 2: Search algorithm from Medline. (DOCX $16 \mathrm{~kb}$ )

Additional file 3: The Study characteristics of included trials about JAK inhibitors. (DOCX 42 kb)

Additional file 4: Results of sensitivity analysis. (TIF $361 \mathrm{~kb}$ )

Additional file 5: Sensitivity analysis of the effect of baricitinib on LDL-C levels (A) and HDL-C levels (B). (DOCX 25 kb)

Additional file 6: Pooled estimates of net change scores of LDL-C with the comparation of JAK inhibitors versus placebo. (TIF $261 \mathrm{~kb}$ )

Additional file 7: Pooled estimates of net change scores of $\mathrm{HDL}-\mathrm{C}$ with the comparation of JAK inhibitors versus placebo. (TIF $240 \mathrm{~kb}$ )

Additional file 8: Risk of bias in the included trials as assessed by the Cochrane risk of bias assessment tool. (DOCX $14 \mathrm{~kb}$ )

\section{Abbreviations}

Cl: Confidence interval; CV: Cardiovascular; DMARD: Disease-modifying antirheumatic drugs; HDL-C: High-density lipoprotein cholesterol; JA: Janus kinases; LDL-C: Low-density lipoprotein cholesterol; LSM: Least squares mean; MACE: Major cardiovascular events; MTX: Methotrexate; RA: Rheumatoid arthritis; RCT: Randomized controlled trial; RR: Risk rations; TC: Total cholesterol; TNF: Tumor necrosis factor; WMD: Weighted mean difference

Acknowledgments

Not applicable.

Availability of data and material

Data are available from the authors on request.

\section{Funding}

This study was supported by the Natural Science Foundation of Hunan Province, China 2017JJ3250.

Authors' contributions

BT conceived and designed the research, CQ and XZ acquired the data, performed statistical analysis, drafted, revised and approved the manuscript submitted. LS and SJ carried out study search and data extraction, ZS and ZD conducted quality assessment; LT and XT performed some data analysis. All authors read and approved the final manuscript.

Ethics approval and consent to participate Not applicable. 


\section{Consent for publication}

Not applicable.

\section{Competing interests}

The authors declare that they have no competing interests.

\section{Publisher's Note}

Springer Nature remains neutral with regard to jurisdictional claims in published maps and institutional affiliations.

\section{Author details}

'Departement of Evidence-base Medcine and Clinical center, The First People's Hospital of Huaihua of University of South China, Huaihua 418000, People's Republic of China. ${ }^{2}$ Department of Pharmacology, The First People's Hospital of Huaihua of University of South China, Huaihua 418000, People's Republic of China. ${ }^{3}$ Department of General Practice, The First People's Hospital of Huaihua of University of South China, Huaihua 418000, People's Republic of China. ${ }^{4}$ Department of Pharmacology, The Second People's Hospital of Huaihua City, Huaihua 418000, People's Republic of China. ${ }^{5}$ Department of Clinical Pharmaccology, Xiangya Hospital Central South University, Changsha 410008, People's Republic of China.

Received: 12 November 2018 Accepted: 5 February 2019

Published online: 18 February 2019

\section{References}

1. Scott DL, Wolfe F, Huizinga TW. Rheumatoid arthritis. Lancet. 2010;376: 1094-108.

2. Burmester GR, Pope JE. Novel treatment strategies in rheumatoid arthritis. Lancet. 2017:389:2338-48.

3. Nakayamada S, Kubo S, Iwata S, Tanaka Y. Recent Progress in JAK inhibitors for the treatment of rheumatoid arthritis. BioDrugs. 2016:30:407-19.

4. Markham A. Baricitinib: first global approval. Drugs. 2017;77:697-704

5. Mullard A. FDA approves Eli Lilly's baricitinib. Nat Rev Drug Discov. 2018:17:460

6. Avina-Zubieta JA, Thomas J, Sadatsafavi M, Lehman AJ, Lacaille D. Risk of incident cardiovascular events in patients with rheumatoid arthritis: a metaanalysis of observational studies. Ann Rheum Dis. 2012;71:1524-9.

7. Matteson EL, Mankad R. Assessment of cardiovascular risk in patients with rheumatoid arthritis. J Rheumatol. 2016:43:1947-9.

8. Westlake SL, Colebatch AN, Baird J, Curzen N, Kiely P, Quinn M, et al. Tumour necrosis factor antagonists and the risk of cardiovascular disease in patients with rheumatoid arthritis: a systematic literature review. Rheumatology (Oxford). 2011:50:518-31.

9. Westlake SL, Colebatch AN, Baird J, Kiely P, Quinn M, Choy E, et al. The effect of methotrexate on cardiovascular disease in patients with rheumatoid arthritis: a systematic literature review. Rheumatology (Oxford). 2010:49:295-307.

10. Lindhardsen J, Gislason GH, Jacobsen S, Ahlehoff O, Olsen AM, Madsen OR, et al. Non-steroidal anti-inflammatory drugs and risk of cardiovascular disease in patients with rheumatoid arthritis: a nationwide cohort study. Ann Rheum Dis. 2014;73:1515-21.

11. Chang $Y$, Robidoux J. Dyslipidemia management update. Curr Opin Pharmacol. 2017;33:47-55.

12. Olsson AG, Angelin B, Assmann G, Binder CJ, Bjorkhem I, Cedazo-Minquez A et al. Can LDL cholesterol be too low? Possible risks of extremely low levels. J Intern Med. 2017;281:534-53.

13. Higgins JJ GS. Cochrane handbook for systematic reviews of interventions. Version 5.1.0 [updated march 2011]. The Cochrane collaboration, 2011 available from wwwcochrane-handbookorg 2011.

14. Higgins JP, Thompson SG. Quantifying heterogeneity in a meta-analysis. Stat Med. 2002;21:1539-58

15. Egger M, Davey Smith G, Schneider M, Minder C. Bias in meta-analysis detected by a simple, graphical test. BMJ. 1997;315:629-34.

16. Begg CB, Mazumdar M. Operating characteristics of a rank correlation test for publication bias. Biometrics. 1994:50:1088-101.

17. Irwig L, Macaskill P, Berry G, Glasziou P. Bias in meta-analysis detected by a simple, graphical test. Graphical test is itself biased. BMJ. 1998:316:470 author reply -1

18. Tanaka Y, Emoto K, Cai Z, Aoki T, Schlichting D, Rooney T, et al. Efficacy and safety of Baricitinib in Japanese patients with active rheumatoid arthritis receiving background methotrexate therapy: a 12-week, double-blind randomized placebo-controlled study. J Rheumatol. 2016:43:504-11.

19. Robertson J, Peters MJ, McInnes IB, Sattar N. Changes in lipid levels with inflammation and therapy in RA: a maturing paradigm. Nat Rev Rheumatol. 2013:9:513-23.

20. Gabay C, Mclnnes IB, Kavanaugh A, Tuckwell K, Klearman M, Pulley J, et al. Comparison of lipid and lipid-associated cardiovascular risk marker changes after treatment with tocilizumab or adalimumab in patients with rheumatoid arthritis. Ann Rheum Dis. 2016:75:1806-12.

21. Chen DY, Chen YM, Hsieh TY, Hsieh CW, Lin CC, Lan JL. Significant effects of biologic therapy on lipid profiles and insulin resistance in patients with rheumatoid arthritis. Arthritis Res Ther. 2015;17:52.

22. Bousoik E, Montazeri Aliabadi H. "do we know Jack" about JAK? A closer look at JAK/STAT signaling pathway. Front Oncol. 2018;8:287.

23. Johnson DE, O'Keefe RA, Grandis JR. Targeting the IL-6/JAK/STAT3 signalling axis in cancer. Nat Rev Clin Oncol. 2018:15:234-48.

24. Li X, Liu ZL, Wu YT, Wu H, Dai W, Arshad B, et al. Status of lipid and lipoprotein in female breast cancer patients at initial diagnosis and during chemotherapy. Lipids Health Dis. 2018;17:91.

25. Semb AG, Rollefstad S, van Riel P, Kitas GD, Matteson EL, Gabriel SE Cardiovascular disease assessment in rheumatoid arthritis: a guide to translating knowledge of cardiovascular risk into clinical practice. Ann Rheum Dis. 2014;73:1284-8.

26. Qiu C, Zhao X, Zhou Q, Zhang Z. High-density lipoprotein cholesterol efflux capacity is inversely associated with cardiovascular risk: a systematic review and meta-analysis. Lipids Health Dis. 2017:16:212.

27. Crowson CS, Matteson EL, Roger VL, Therneau TM, Gabriel SE. Usefulness of risk scores to estimate the risk of cardiovascular disease in patients with rheumatoid arthritis. Am J Cardiol. 2012;110:420-4

28. Taylor PC, Keystone EC, van der Heijde D, Weinblatt ME, Del Carmen Morales L, Reyes Gonzaga J, et al. Baricitinib versus placebo or adalimumab in rheumatoid arthritis. N Engl J Med. 2017;376:652-62.

29. Fleischmann R, Schiff M, van der Heijde D, Ramos-Remus C, Spindler A, Stanislav M, et al. Baricitinib, methotrexate, or combination in patients with rheumatoid arthritis and no or limited prior disease-modifying Antirheumatic drug treatment. Arthritis Rheumatol. 2017;69:506-17.

30. Dougados $M$, van der Heijde D, Chen YC, Greenwald M, Drescher E, Liu J, et al. Baricitinib in patients with inadequate response or intolerance to conventional synthetic DMARDs: results from the RA-BUILD study. Ann Rheum Dis. 2017:76:88-95.

31. Genovese MC, Kremer J, Zamani O, Ludivico C, Krogulec M, Xie L, et al. Baricitinib in patients with refractory rheumatoid arthritis. N Engl J Med. 2016;374:1243-52

32. Tanaka Y, Ishii T, Cai Z, Schlichting D, Rooney T, Macias W. Efficacy and safety of baricitinib in Japanese patients with active rheumatoid arthritis: a 52-week randomized, single-blind, extension study. Mod Rheumatol. 2018;28:20-9.

33. Keystone EC, Taylor PC, Drescher E, Schlichting DE, Beattie SD, Berclaz PY, et al. Safety and efficacy of baricitinib at 24 weeks in patients with rheumatoid arthritis who have had an inadequate response to methotrexate. Ann Rheum Dis. 2015:74:333-40.

Ready to submit your research? Choose BMC and benefit from:

- fast, convenient online submission

- thorough peer review by experienced researchers in your field

- rapid publication on acceptance

- support for research data, including large and complex data types

- gold Open Access which fosters wider collaboration and increased citations

- maximum visibility for your research: over $100 \mathrm{M}$ website views per year

At BMC, research is always in progress.

Learn more biomedcentral.com/submissions 